http://jmscr.igmpublication.org/home/

ISSN (e)-2347-176x ISSN (p) 2455-0450

crossref DOI: https://dx.doi.org/10.18535/jmscr/v8i2.132

\title{
Estimation of Cardiovascular Risk in an Individual Patient without a Known Cardiac Disease in General Medicine Department of Aditya Multi Speciality Hospital, Guntur
}

\author{
Authors \\ Pallaprolu. Jahnavi ${ }^{1 *}$, Yarneni. Alekhya ${ }^{2}$, Korivi. Anand ${ }^{3}$, \\ Hima Vamsi. Govindu ${ }^{4}$, Dr Chirumella. Ajay Babu ${ }^{5}$ \\ ${ }^{1,2,3,4}$ Student, ${ }^{5}$ Professor \\ Department of Pharmaceutics, M.A.M College of pharmacy, \\ Kesanupalli, Narasaraaopet, Pin: 522601, Guntur (Dt) Andhra Pradesh, India \\ *Corresponding Author \\ Jahnavi. Pallaprolu
}

\begin{abstract}
Cardiovascular system consists of three interrelated components such as blood, heart and blood vessels. Cardiovascular diseases are those which affect the structure or functioning of heart and blood vessels involved. Cardiovascular diseases are the most common cause of death globally and leading cause of death in India meanwhile. The objective of this is to determine the patient's individual risk of getting cardiovascular disease. The risk of an individual person is determined by using QRISK3-2018 risk score. This study is performed to assess the cardiovascular risk for the next 10 years in an individual patient without known cardiac disease. A prospective study is conducted in general medicine department of Aditya Multi Speciality hospital, Guntur. This study uses the updated QRISK3-2018 risk score method to analyze the person risk of getting cardiovascular risk. This study included patients of above 25years and below 65 years. By observing the results obtained, it is found that increased cardiovascular risk is observed in patients with increased age and having diabetes mellitus along with hypertension.
\end{abstract}

Keywords: Cardiovascular disease, risk factors, QRISK3-2018 risk score.

\section{Introduction}

Cardiovascular system consists of three major components involving blood, the heart and blood vessels. $^{[1]}$ Heart diseases mainly affect the structure of functioning of heart and blood vessels including blood vessel diseases, arrhythmias, heart defects, heart muscle disease, and heart valve disease. ${ }^{[2]}$ CVDs are the number one cause of death globally and also the leading cause of death in India. Many surveys showed that rising prevalence of major risk factors for CVD in Asian
Indian population is increasing. Due lack of surveillance system and proper diagnosis in India, the risk of developing cardiovascular diseases is getting increased. Here, this study helps us to point out the need of research for developing the proper diagnosis system of CVDs. ${ }^{[3]}$

When modifiable risk factors are treated and corrected, the chances of getting CVD will also reduce. ${ }^{[4]}$

The three main functions of cardiovascular system are transportation of materials, protection from 
pathogens, and regulation of the body homeostasis.

\section{Cardiovascular Disease}

Cardiovascular disease (CVD) is a general term for affecting the heart or blood vessels usually associated with building of fatty deposits inside the arteries called atherosclerosis and causes increased risk of blood clots. It is also associated with damage to arteries in specific organs such as the brain, heart, eyes and kidneys. ${ }^{[5]}$ Sometimes cardiovascular disease is also called as heart disease, but they are not exactly same in medical terms. Where, heart disease is a general term for conditions which are affecting the structure of heart and functioning of heart. All heart diseases are cardiovascular disease but not all cardiovascular diseases are heart diseases. ${ }^{[6]}$

\section{Types of cardiovascular disease}

There are different types of CVD

- Coronary Heart Disease: coronary heart disease is sometimes called ischemic disease. CHD occurs when the flow of blood which is rich in oxygen to heart muscle gets blocked or reduced. The most common symptoms of CHD are angina, heart attack, heart failure.

- Stroke: It is a serious life-threatening condition which occurs when the blood supply to the part of brain is reduced. This condition is medical emergency and urgent treatment is necessary. As soon as person receives treatment for stroke, it causes less damage. Two main causes of stroke are ischemic and hemorrhagic stroke.

- Transient Ischemic Attack (TIA): TIA or mini stroke caused by temporary disruption of blood supply to part of brain. This can cause sudden symptoms which are similar to stroke.

- Peripheral Arterial Disease: PAD which build-up fatty deposits in the arteries which restrict blood supply to leg muscles.
It is also known as peripheral vascular disease.

- Aortic Disease: aortic diseases are a group of conditions affecting aorta which is the largest blood vessel in the body it carries blood from heart to rest of the body.

- Abnormal heart rhythm: An irregular or abnormal heart beat is called an arrhythmia.

- Heart valve disease: Valves sit at the exit of four heart chambers and maintains oneway blood flow through your heart. Heart valve problems include mitral valve prolapse, aortic stenosis, Mitral valve insuffiency.

- Congenital Heart Disease: Defect of one or more structures of heart or blood vessels.

- Cardiomyopathy: disease of heart muscles.

- Pericarditis: inflammation of lining surrounding the heart.

\section{Cardiovascular Risk Factors}

There are many risk factors some can be controlled and others can't be controlled.

\section{Modifiable risk factors}

a) Physical inactivity: it causes increase of heart diseases. If you increase physical activity that protects you by regulating your body weight, and increases the use of body's insulin. Stress also increases the cardiovascular risk factors. So by doing physical activity like running, or riding bike cause stress relief which further cause decreased cardiovascular disease.

b) Tobacco: smoking promotes cardiovascular disease by damaging the endothelium, increasing fatty deposits in arteries, increases in clotting, and raises LDL, and reduces HDL. So by reducing smoking maintains good health of heart and decrease risk of cardiovascular disease.

c) Diet: Diet plays a major role in development or prevention of cardiovascular disease. A 
diet high in fatty acids cause increased risk in cardiovascular disease. So by maintaining healthy diet by intake of foods containing low saturated fat, and plenty of fresh fruits and vegetables.

d) Bad fats: High intake of bad fats causes raise in blood cholesterol, which blocks arteries, and increases risk of heart disease.

e) Blood lipids: abnormal levels of lipids cause increase in heart disease.

f) Hypertension: this stress your body's blood vessel, causes blood clots in arteries and makes them weaken. This leads to atherosclerosis and narrowing of blood vessels and makes them more likely to block blood vessels.

g) Obesity: Overweight causes hypertension, diabetes and atherosclerosis, which causes increased risk of cardiovascular disease. If BMI increases it propose increase in cardiovascular risk. ${ }^{[7]}$

h) Diabetes mellitus: it also increases the risk of heart disease. As body needs energy to maintain energy it is done via using glucose. Insulin is a hormone produced by pancreas which helps to move blood glucose from food to body cells. If person is with diabetes mellitus then body doesn't make enough insulin.

\section{Non-modifiable risk factors:}

a) Family history: type 2 diabetes also has a genetic component, so if your parents are having diabetes then you are having increased risk of developing it too. As type

2 diabetes mellitus is also a cause of developing cardiovascular disease.

b) Age: getting old is also a risk factor of cardiovascular disease.

c) Gender

d) Ethnicity

e) Socioeconomic status

\section{Objectives of Work}

To raise awareness of the symptoms and severity of heart diseases among the general public by examining the prevalence of heart attack or stroke and their association with cardiovascular, Where as identifying the factors responsible for the epidemic of chronic heart failure in the ageing population, and to identify potential areas of prevention. To elucidate reasons for regional variations in CVD morbidity and mortality by assessing the patient quality of life. And to evaluate the percentage of risk in unknown cardiac patients by estimating the major risk factors causing increased risk of cardiac disorders and finally to maintain the healthy outcome by avoiding the possible risk factors.

\section{Methods}

\section{Materials and Methods}

A prospective observational study was conducted at general medicine department of Aditya Multi Speciality Hospital, Guntur. This study included the patients without known cardiovascular disease aged between 25 to 65years. The $10 \mathrm{yr}$ cardiovascular risk for every patient was calculated by using the QRISK score and the risk was categorized as high risk, moderate risk and low risk.

\section{Study Site}

This study was conducted at Aditya Multi Speciality Hospital, Guntur. The different specialties of this hospital including departments like general medicine and orthopedic department.

\section{Study Centre}

This observational study was carried out in general medicine inpatient and outpatient department of Aditya Multi Speciality Hospital, Guntur.

\section{Study Design}

This study was prospective observational study. Prospective study is that which watches outcomes which are obtained by development of disease during the study period. This stud usually involves cohort subjects and determines outcome by watching over a long period. The outcome should be common, or the number of outcomes observed will be too small to be statistically meaningful. 


\section{Study Duration}

This study was carried out for 6 months.

\section{Study Population}

Patients who are attending general medicine department of Aditya Multi Speciality Hospital, Guntur.

\section{Sample Size}

Total number of patients enrolled in this study is 200.

\section{Patient Selection and Procedure Study Criteria \\ Inclusion Criteria}

* Patients with age group of 25 to 65 years are included.

* Inpatients and outpatients of general medicine department are included.

* Patients with complete information are included

* Patients with any chronic or acute disease are included.

\section{Exclusion Criteria}

* Patients with age group of $<25$ and $>65$ are excluded.

* Pediatrics and pregnancy population are excluded.

* Patients with incomplete information are excluded.

* Patients with known cardiac diseases are excluded.

\section{QRISK3 web calculator}

To evaluate the risk for the obtained subjects we have performed by using the QRISK 3 - 2018 web calculator. The QRISK $^{\circledR} 3$ algorithm calculates a person's risk of developing a heart attack or stroke over the next 10 years. It presents the average risk of people with the same risk factors as those entered for that person. The QRISK $^{\circledR} 3$ algorithms was developed by doctors and academics working in the UK National Health Service and it is based on routinely collected data from many thousands of GPs across the country who has freely contributed data to the Q Research database for medical research.

QRISK $^{\circledR} 3$ has been validated on a separate group of practices from that used to develop the score and the performance is very good.

\section{Study Procedure}

The visit was done at the department of general medicine department.

Patient data was collected in the pre-designed data collection form. The data collection form includes demographics, patient history of disease and other co-morbidities including the study. The sufficient data was collected from the patient by asking the involved questions without hurting them. All the collected information should be clearly entered in the particular questionnaire form involved. Should clearly get information from respective patients without any doubt for further analysis to be performed. The total sample collected for the study is 200 from inpatient and outpatient department of general medicine in Aditya multi Speciality hospital, Guntur. The collected data are separated as per age, gender, and including some other co-morbidities. To evaluate the risk for the obtained subjects are performed by using the QRISK 3 - 2018 web calculator. The QRISK $^{\circledR} 3$ algorithm calculates a person's risk of developing a heart attack or stroke over the next 10 years. The QRISK score was calculated individually for the patient involved. It presents the average risk of people with the same risk factors as those entered for that person. The obtained QRISK value was noted accordingly and then it was compared with the involved co-morbidities by using the statistical analysis method. The statistical analysis of the collected data was performed by using SPSS software (Statistical Package for the Social Sciences). By using Pearson correlation method in SPSS software, QRISK score was compared with co-morbidities involved. The patient's data was analyzed and categorized and then correlated.

\section{Statistical Analysis}


Statistical analysis was performed by using MS Excel, and the results were statistically analyzed by using the Pearson correlation method in SPSS software by comparing the co-morbidities involved.

\section{Correlation}

Sometimes two continuous characters are measured in the same person, such as weight and cholesterol, weight and height, etc. The relationship or association between two quantitatively measured or continuous variables is called correlation.

The extent of correlation varies between minus one and plus one, i.e. $-1<\mathrm{r}<1$.

\section{Pearson correlation coefficient}

Correlation is a measure of two sets of data to know how well they are related. Pearson correlation is the most common measure of correlation in statistics. The full name is the Pearson Product Moment Correlation (PPMC). Pearson correlation shows linear relation between two sets of data.

At 0.05 , level of significance is chosen. When the p-value is low, it means that the observed values are significantly different from the population values that were hypothesized in the beginning. The p-value is said to be more significant, if it is as lower as possible. If the P-value is less, the obtained result will be defined as more significant. But, getting a P-value less than 0.05 is quite a less common practice.

\section{Results}

This is a prospective observational study of patients referred to the department of general medicine at Aditya multi Speciality hospital, Guntur, who comes for treatment of various conditions of diseases and general follow up from October 2018 to February 2019.

The selected sample was analyzed in 3 different parts

PART1: Demographical analysis

PART 2: QRISK determination based on age and gender

PART 3: Determining the co-morbidities using Pearson Correlation coefficient

\section{Part 1: Demographical Analysis}

The socio demographic characters included in this study are age, gender, smoking, and diabetes mellitus, Chronic Kidney Disease, Blood Pressure, Migraine and Rheumatoid Arthritis.

And the total sample collected during the duration of study is 200 , i.e., $\mathrm{N}=200$.

Table 1: Demographical Analysis

\begin{tabular}{|l|c|c|c|}
\hline \multicolumn{2}{|c|}{ SOCIODEMOGRAPHICS } & $\begin{array}{c}\text { NO.OF } \\
\text { SUBJECTS }\end{array}$ & $\begin{array}{c}\text { PERENTAGE } \\
(\%)\end{array}$ \\
\hline \multirow{4}{*}{ AGE } & & 78 & 39 \\
\cline { 2 - 4 } & $25-34$ & 160 & 80 \\
\cline { 2 - 4 } & $35-44$ & 31 & 15.5 \\
\cline { 2 - 4 } GENDER & $45-55$ & 23 & 11.5 \\
\hline \multirow{3}{*}{ SMOKING } & $55-64$ & 125 & 62.5 \\
\cline { 2 - 4 } & MALE & 75 & 37.5 \\
\cline { 2 - 4 } & FEMALE & 114 & 57 \\
\cline { 2 - 4 } & EX SMOKERS & 11 & 5.5 \\
\cline { 2 - 4 } & MODERATE & 31 & 15.5 \\
\cline { 2 - 4 } & HEAVY & 19 & 9.5 \\
\hline \multirow{3}{*}{ DIABETES MELLITUS } & YES & 22 & 11 \\
\hline \multirow{3}{*}{ CHRONIC KIDNEY DISEASE } & NO & 80 & 60 \\
\hline \multirow{3}{*}{ BLOOD PRESSURE } & YES & 120 & 14.5 \\
\hline \multirow{3}{*}{ MIGRAINE } & NO & 168 & 84 \\
\hline & YES & 80 & 40 \\
\cline { 2 - 4 } & NO & 120 & 60 \\
\cline { 2 - 4 } & YES & 29 & 14.5 \\
\cline { 2 - 4 } & NO & 171 & 85.5 \\
\hline
\end{tabular}




\begin{tabular}{|c|c|c|c|}
\hline RHEMATOID ARTHRITIS & NO & 161 & 80.5 \\
\hline \multicolumn{2}{|c|}{ TOTAL } & $\mathrm{N}=200$ & \\
\hline
\end{tabular}

\section{Part 2 QRISK Determination Based On Age and Gender}

Chart 2: Age Distribution among the Study Sample (with respect to risk determined)

\section{AGE DISTRIBUTION}

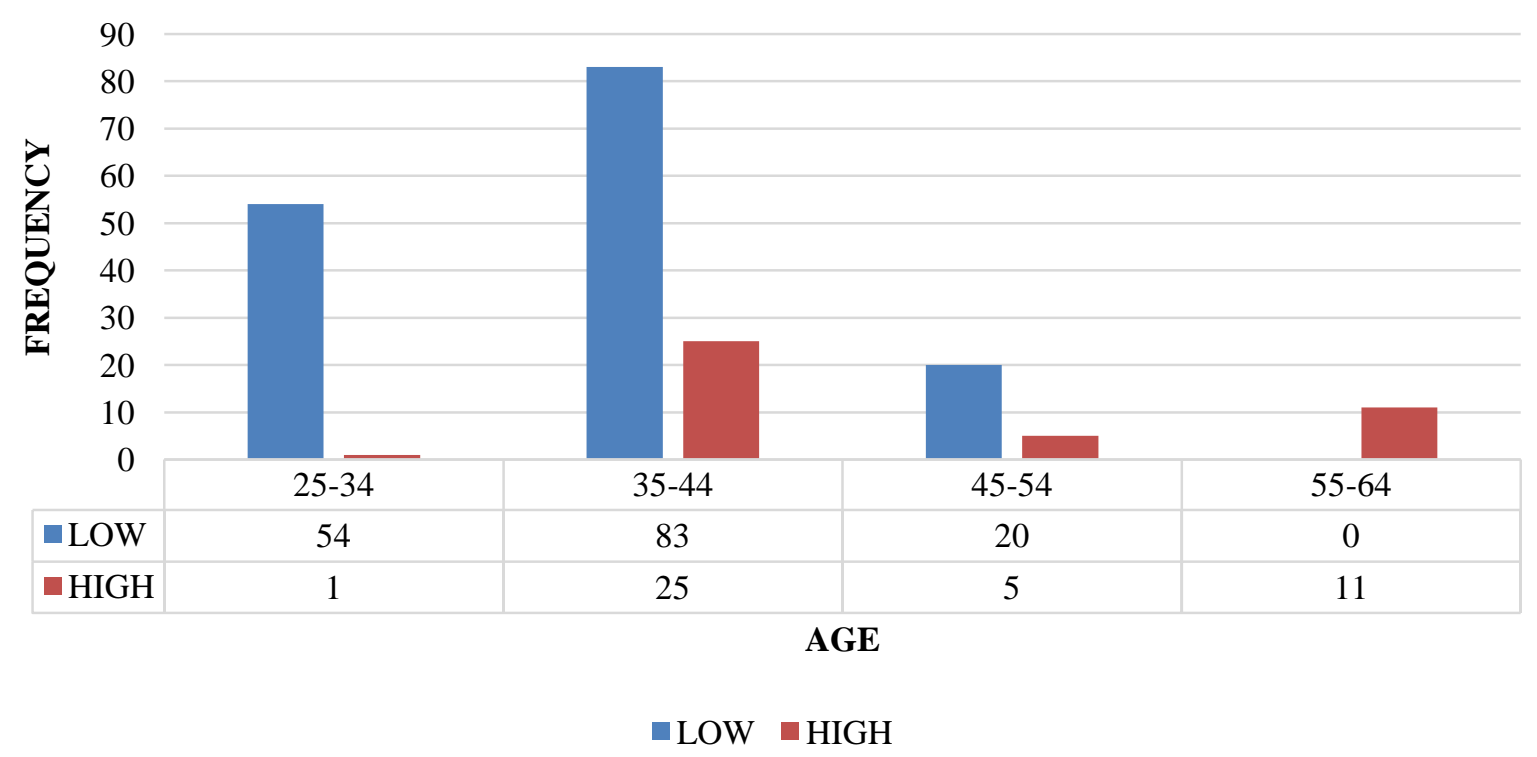

Based on the collected samples, age has been divided into four groups including 10 subjects per group and risk is calculated by determining low and high risk of involved patients. By observing the above data, age group of increased age is having high risk of getting cardiovascular disease when compared to other age groups.

Chart 3: Gender Distributions among the Study Sample (With respect to risk determined)

\section{GENDER DISTRIBUTION}

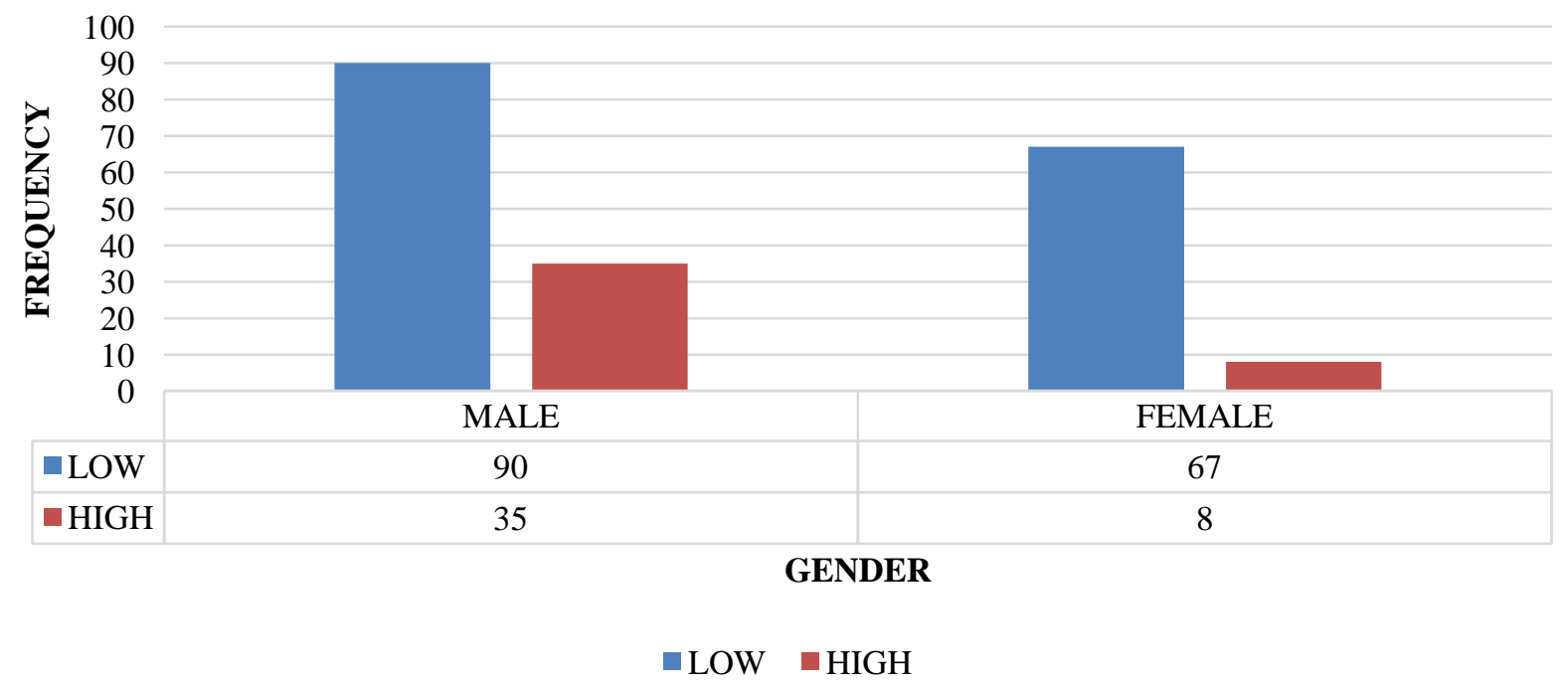


Based on the collected samples gender variance has shown from the above bar diagram by determining the low and high risk. When

\section{Part 3 Correlation Methods for two Different Parameters}

Table 2: Pearson's Correlation of Age and Q Score compared to males and females, the observed result shows increased risk in males

Pearsons Correlations of age and $Q$ score

\begin{tabular}{|ll|r|r|}
\hline & & \multicolumn{1}{|c|}{ AGE } & Q score \\
\hline AGE & Pearson Correlation & 1 & $.445^{\mathrm{Nx}}$ \\
& Sig. (2-tailed) & & .000 \\
& $\mathrm{~N}$ & 200 & 200 \\
\hline O score & Pearson Correlation & $.445^{\mathrm{k} x}$ & 1 \\
& Sig. (2-tailed) & .000 & \\
& $\mathrm{~N}$ & 200 & 200 \\
\hline
\end{tabular}

*. Correlation is significant at the 0.01 level (2-tailed).

Here Pearson correlation was done between age and Q score obtained from the collected data. Where, Pearson correlation was explained by positive correlation and negative correlation. At level of significance +1 , we got the positive correlation of the value of 0.445 .
Where, the significance value i.e., the P-value which shows positive value at less than 0.05 . Here we got $\mathrm{P}$-value of 0.000 where it determines the positive $\mathrm{P}$-value.

Table 3 Pearson Correlations of DM and HTN with Q Score

Pearson Correlations of DM and HTN with Q score

\begin{tabular}{|ll|r|r|}
\hline & & Q score & DM AND HTN \\
\hline Q score & Pearson Correlation & 1 & $.258^{\prime \prime}$ \\
& Sig. (2-tailed) & & .000 \\
& N & 200 & 200 \\
\hline DM AND HTN & Pearson Correlation & $.258^{\prime \prime \prime}$ & 1 \\
& Sig. (2-tailed) & .000 & \\
& $N$ & 200 & 200 \\
\hline
\end{tabular}

*. Correlation is significant at the 0.01 level (2-tailed).

Correlation was done between combined Diabetes

mellitus and hypertension subjects with 
comparing Q score. Here, positive correlation has obtained at the value of 0.258 in Diabetes and hypertensive subjects at level of significance.

Significant value of less than 0.05 i.e., the obtained value is 0.000 has obtained and shows positive response.

\section{Discussion}

Based on the observed results, we have noticed that increase in age causes increase in cardiovascular diseases. Males are more affected to cardiovascular risk factors when compared to females. Increase in age causes decrease in physiological activity of body organs and leads to drug toxicity. Males with smoking habit are having increased risk. Subjects with diabetes and hypertension will have affect in the subject's blood glucose level and will have increased pressure in arteries which causes sudden increase in blood pressure leads sudden cardiac arrest.

In this study, increased cardiac diseases are observed mainly due to increase in risk factors of diabetes and hypertension with respective to age. By doing statistical analysis of diabetes and hypertensive subjects, the Pearson value is noted at less than 1 which shows positive result of increased risk in respective subjects. As well as in age parameter with QRISK score it shows positive result. Here persons with increased age, and who are not maintaining a good life style are more prone to have increased cardio vascular diseases.

Diabetes and hypertension has become the leading cause of disease in worldwide, many are having this primary co morbidity at early age of their life span and causes much risk towards. Managing diabetes and hypertension plays a major role in maintaining cardiovascular diseases.

- Other co morbidities like rheumatoid arthritis, migraine, chronic kidney diseases are identified in less number of subjects. As well the subjects with such conditions are on medication with prescription which shows good affect on their life disease.

- Here results were drawn only basing age and gender along with diabetes and hypertension as they plays a primary role in increase of cardiovascular risk.

- So we have not accounted the other co morbidities while performing the results due to assumption of having less risk of having cardiovascular diseases.

- When subjects having hypertension, their systolic blood pressure plays a major role in cardiovascular diseases as it is an increased pressure in arteries during the contraction of heart muscle.

- Subjects who are on OTC medication cause risk of cardiovascular disease, as due to intake of medication without caution. So in order to avoid risk of cardiovascular system patients or individuals should take prescribed medication.

Finally, the obtained results show that increase in cardiovascular disease is directly proportional to increase in age and diabetes and hypertension. When diabetes and hypertension measured individually it shows less risk when compared to combined diseased individuals.

\section{Conclusions}

The present study aimed to evaluate the cardiovascular risk in an individual patient without a known cardiac disease by using QRISK3 calculator. Here we included the subjects of age 25 to 65 years to check that who are having increased risk of cardiovascular risk in an individual patient for the next 10 years.

Here we finally conclude that patients with increased age are having high risk of cardiovascular disease. So, elderly patients needs regular checkup. It shows their health status and stage of disease and it helps to maintain a good treatment and life style.

Patients with a known diabetes and hypertension should maintain a healthy diet and physical activity which reduce their disease effect on other systems of body. By estimating the risk in individuals of diabetes and hypertension shows easy way to counsel them about their disease and 
their medication and produce a chance to reduce their illness.

Patients with increased age have decreased metabolism and clearance when compared to adults, and this causes decrease in drug clearance and leads to toxicity of such drug. So in elderly patients the proper counseling should be needed to avoid such kind of risk. And while prescribing drugs in elderly patients they should check all the lab values and should note their renal function. Based on this renal and liver functioning drugs should be prescribed with a definite dosage and dosing intervals.

In order to avoid further risk in diabetes mellitus and hypertensive patients, medication adherence and patient compliance is important. People with diabetes and hypertension should maintain a healthy heart based on their activities and should maintain a normal value of blood glucose and BP levels.

\section{References}

1. Principles of Anatomy and Physiology 13ed - G. Tortora, B. Derrickson (Wiley, 2012) BBS, Page No 728.

2. Journal Of Cardiovascular Diseases \& Diagnosis, Index Copernicus Value: 82.47, NLM ID: 101613243

3. Cardiovascular Disease Risk Factors in Asian Indian Population: A Systematic Review, Tanmay Nag, Arnab Ghosh. Journal Of Cardiovascular Disease Research, Volume 4, Issue 4, December 2013

4. Cardiovascular Disease Risk Assessment: Insight From Framingham, Ralph B. D’Agostino, Et Al, Journal Of Global Heart, 2013 March: 8(1), PMC3673738

5. Cardiovascular Disease Conditions, National Health Service-UK, Causes Of CVD

6. National Heart, Lung and Blood Institute. "Know The Differences:
Cardiovascular Disease, Heart Disease, And Coronary Heart Disease.” Accessed August 7, 2018.

7. Risk Factors, World Heart Federation, 30 May 2017. 